\title{
Simulação unidimensional da transferência de calor na articulação do joelho canino durante os processos de aquecimento e de resfriamento terapêuticos
}

\author{
Fernanda Souza da Silva*, Adriana Silva França, Marcos Pinotti
}

\begin{abstract}
Resumo A aplicação terapêutica de calor e de frio é amplamente utilizada na prática clínica da fisioterapia, sendo especialmente relevante no tratamento de lesões do sistema músculo esquelético. Para que os benefícios decorrentes dessas terapias possam ser alcançados é necessário, entretanto, que o tecido alvo atinja limites ótimos de temperatura. Temperaturas fora desse limite podem promover tratamentos ineficazes ou dano tecidual. Compreender o comportamento da temperatura tecidual em resposta às aplicações térmicas é, portanto, essencial para que esses tratamentos sejam seguros e eficazes. Portanto, este estudo teve como objetivo simular a transferência de calor nos tecidos que compõem a articulação do joelho canino durante o aquecimento e o resfriamento terapêuticos. Os resultados obtidos foram comparados com dados experimentais in vivo. A articulação do joelho canino foi representada por um cilindro composto por seis camadas teciduais. A transferência de calor transiente na direção radial foi avaliada com base no modelo de Pennes. Foram avaliados dois tipos de condições de contorno: i) temperatura conhecida na superfície externa (aquecimento e resfriamento) e ii) fluxo de calor conhecido na superfície externa (aquecimento). As simulações baseadas na pressuposição de que temperatura na superfície externa era equivalente à temperatura do dispositivo de aquecimento (A1) e de resfriamento (R1) não apresentaram uma descrição satisfatória das medidas experimentais, com diferenças percentuais médias de $14,8 \%$ e $17,1 \%$ para o aquecimento e resfriamento, respectivamente. Obteve-se boa concordância com os dados experimentais (diferença percentual $<2 \%$ ) para as simulações baseadas nos valores conhecidos da temperatura da epiderme (A2) e do fluxo de calor na superfície (A3). Os resultados obtidos demonstraram que o modelo unidimensional proposto no presente estudo é apropriado para a avaliação das temperaturas médias teciduais durante o processo de aquecimento, porém não tão adequado para simular o processo de resfriamento.
\end{abstract}

Palavras-chave Biotransferência de calor, Equação de Pennes, Simulação computacional, Aquecimento terapêutico, Resfriamento terapêutico, Elementos finitos.

\section{One-dimensional simulation of heat transfer in the canine knee joint during therapeutic heating and cooling}

\begin{abstract}
Therapeutic application of heat and cold is commonly used in physical therapy, being specially relevant to the treatment of lesions of the muscular and skeletal systems. However, for the benefits of this therapy to be achieved, it is necessary for the therapeutic target to be heated or cooled within specified temperature limits. Correct assessment of tissue temperature is therefore an important strategy for safe and effective treatments. Thus, this study aimed at the simulation of the transient heat transfer taking place in the canine knee, resulting from the application of therapeutic heating and cooling. The results obtained were compared to in vivo experimental data. The canine knee joint was represented by a cylinder comprised of six layers. Transient heat transfer in the radial direction was evaluated based on the model proposed by Pennes. Simulations were performed employing two types of boundary conditions at the external joint surface: i) known temperature (heating/cooling) and ii) known heat flux (heating). Simulations based on the assumption that the external surface temperature was equivalent to that of the heating (A1) and cooling (R1) device did not present a satisfactory description of the experimental data, with average percent differences of $14.8 \%$ and $17.1 \%$ for heating and cooling, respectively. There was good agreement with experimental data (average percent differences $<2 \%$ ) for simulations based on known temperatures of the epidermis (A2) or known heat flux at the surface (A3).The obtained results showed that the one-dimensional model proposed in the present study is appropriate for evaluation of average tissue temperatures during heating, but less adequate for cooling simulations.
\end{abstract}

Keywords Bioheat transfer, Pennes equation, Computational simulation, Therapeutic heating, Therapeutic cooling, Finite element method.

*e-mail: nandasouzafisio@yahoo.com.br Recebido: 07/04/2011 / Aceito: 12/08/2011 


\section{Extended abstract}

\section{Introduction}

Therapeutic application of heat and cold is commonly used in physical therapy, being specially relevant to the treatment of lesions of the muscular and skeletal systems (bones, ligaments, muscle and tendons). However, for the benefits of this therapy to be achieved, it is necessary for the therapeutic target to be heated or cooled within specified temperature limits. Correct assessment of tissue temperature is therefore an important strategy for safe and effective treatments. Even though in vivo tissue temperature determination is possible, there are several risks and difficulties in this type of procedure given its invasive character, lack of precision and control of several parameters and measurement limitations. Thus, this study aimed at the simulation of the transient heat transfer taking place in the canine knee joint, resulting from the application of therapeutic heating and cooling. Heat transfer was evaluated based on the bioheat transfer model proposed by Pennes, employing a software based on the finite element method.

\section{Material and Methods}

Software verification was based on a one-dimensional, stationary bioheat transfer problem in cartesian coordinates considering one layer of muscle tissue. The simulation domain (geometric model of the canine knee) was defined by a cylinder comprised of six tissue layers, from the outside to the inside: epidermis; subcutaneous tissue; fat; muscle; pericapsular region; and intra-articular cavity (including bone and synovial fluid). The symmetry boundary condition $(q=0)$ was applied at the center of the joint $(r=0)$. Two types of boundary conditions were evaluated for the external epidermis surface: i) temperature equivalent to the average temperature of the heating/cooling device; ii) temperature equivalent to the average locally measured value after stabilization; and iii) heat flux at the surface corresponding to the average heat flux provided by the heating device. Simulation results were compared to in vivo experimental measurements.

\section{Results and Discussion}

Verification tests indicated that the employed software provides accurate calculations of the bioheat transfer (Pennes) equation. A comparison of the simulations and experimental data showed that there were significant differences between experiments and simulations (14.8\% and $17.1 \%$ average differences for heating and cooling, respectively) when the surface temperature was considered to be equivalent to the average temperature of the heating/cooling device. Overall the boundary condition based on heat flux provided a better description of the heat transfer phenomena that takes place during therapeutic heating, with a significant reduction in differences between experiments and simulations (2.1\% average difference). Further studies will target model improvements by including real variations of the knee joint geometry (two-dimensional simulations).

\section{Conclusion}

This study simulated the heat transfer in the canine knee joint during heating and cooling treatments. The simulations $A 2$ and $A 3$ represent adequately the heating process. However results indicate that the computer simulation, considering the boundary condition of heat flux can be an interesting alternative to evaluate the effectiveness of heat during heating process. Simulations of cooling did not show good approximation with the experimental data. The simulations in this study are the first step to a better understanding of the behavior of non-invasive tissue temperature during application of physical heating and cooling. 


\section{Introdução}

Os recursos térmicos de aquecimento e de resfriamento, em diversas formas, são utilizados com finalidades terapêuticas desde os tempos antigos (Doherty et al., 2009). Atualmente, esses recursos são amplamente empregados na prática clínica da fisioterapia, principalmente para o tratamento de lesões do sistema músculo esquelético: osso, ligamento, músculos e tendões (Nadler et al., 2004; Zhang et al., 2008). Para que os efeitos terapêuticos decorrentes da aplicação de calor ou de frio sejam obtidos, é necessário, entretanto, que o tecido alvo atinja uma faixa ótima de temperatura (Draper et al., 1999).

Estudos preconizam que para que os efeitos terapêuticos decorrentes da diminuição da temperatura ocorram, é necessário que os tecidos atinjam a faixa de $13,0{ }^{\circ} \mathrm{C}$ a $18,3{ }^{\circ} \mathrm{C}$ (Chesterton et al., 2002). Alguns trabalhos sugerem também que para assegurar a integridade dos tecidos profundos, é interessante que temperatura da pele seja considerada como referência e mantenha-se entre $10^{\circ} \mathrm{C}$ a $15^{\circ} \mathrm{C}$ (Deal et al., 2002; Greenstein, 2007; Kennet et al., 2007). No aquecimento, é recomendado que por pelo menos 5 minutos a temperatura do tecido alvo encontre-se na faixa de $40{ }^{\circ} \mathrm{C}$ a $45^{\circ} \mathrm{C}$ (Draper et al., 1999; Robertson et al., 2005).

De acordo com Maggi et al. (2008), valores fora da faixa terapêutica recomendada podem ocasionar tratamentos insuficientes para produzir os efeitos terapêuticos desejados ou dano térmico ao tecido, com consequente destruição do citoesqueleto, da membrana celular e da microvasculatura regional. Tais lesões podem causar interrupção do ciclo reprodutivo celular e do fluxo sanguíneo no local, o que predispõe à necrose tecidual (Knight et al., 2001).

Desta forma, o sucesso das terapias localizadas de frio e de calor é altamente dependente da compreensão do comportamento da temperatura e do processo térmico que ocorre no interior dos tecidos alvo e adjacente (Karaa et al., 2005). Embora determinações in vivo da temperatura tecidual possam ser empregadas com esse objetivo, ainda existem grandes dificuldades e riscos associados à realização desses procedimentos, principalmente, pelo caráter invasivo, pela imprecisão no controle de diversos parâmetros e pelas limitações das medidas (Trobec et al., 2008; Shrivastava e Vaughan, 2009).

Atualmente, um grande número de ferramentas numéricas e computacionais da engenharia tem sido utilizado para conhecer e interpretar os processos e fenômenos biológicos. Dentre estas ferramentas, as simulações numéricas têm sido de grande importância em uma gama de problemas relacionados, em particular, à transferência de calor nos tecidos vivos. Tal instrumento pode colaborar fortemente para a análise e orientação dos procedimentos térmicos realizados pelos profissionais da saúde, permitindo que os mesmos atinjam seus objetivos de forma eficaz e segura.

Diante do exposto, o presente trabalho teve como objetivo simular a transferência de calor na articulação do joelho canino, aproximando a geometria do joelho por um cilindro (simulação unidimensional), durante os processos de aquecimento e resfriamento terapêuticos.

\section{Materiais e Métodos}

A equação de biotransferência de calor de Pennes (1948) com o termo de perfusão sanguínea foi adotada para a determinação da distribuição da temperatura dos tecidos que compõem a articulação do joelho canino. Esta equação pode ser descrita como:

$\rho c_{p} \frac{\partial T}{\partial t}=\nabla(k \nabla T)+(\rho \omega)_{s} c_{p s}\left(T_{a}-T\right)+q_{m e t}$

em que: $k$, r e $c_{\mathrm{p}}$ representam a condutividade térmica $\left[\mathrm{W} / \mathrm{m} \cdot{ }^{\circ} \mathrm{C}\right]$, a massa específica $\left[\mathrm{kg} / \mathrm{m}^{3}\right]$ e o calor específico do tecido $\left[\mathrm{J} / \mathrm{kg} \cdot{ }^{\circ} \mathrm{C}\right]$, respectivamente. $c_{\mathrm{ps}}$ é o calor específico do sangue; $\mathrm{r}_{\mathrm{s}}$ correspondente a massa específica do sangue; $w_{\mathrm{s}}$ é a perfusão sanguínea; $\mathrm{q}_{\mathrm{met}}$ é a geração de calor metabólico; $\mathrm{T}$ é a temperatura do tecido $\left[{ }^{\circ} \mathrm{C}\right]$ e $T_{\mathrm{a}}$ é a temperatura arterial $\left[{ }^{\circ} \mathrm{C}\right]$, considerada como $37^{\circ} \mathrm{C}$.

Os estudos de simulação foram efetuados pelo software FEHT (F-Chart Software, Madison, WI), baseado na técnica de elementos finitos.

A verificação do software FEHT foi realizada previamente a realização das simulações unidimensionais da transferência de calor na articulação do joelho. A verificação foi baseada em um problema unidimensional de biotransferência de calor, com solução analítica possível, envolvendo somente a camada de tecido muscular. Esse tecido foi escolhido devido à alta perfusão sanguínea em relação às demais estruturas e tecidos articulares. Segundo Cui e Barbanel (1990), a perfusão sanguínea é um dos principais parâmetros que influenciam o comportamento da temperatura dos tecidos biológicos.

Duas situações distintas foram consideradas: aquecimento, em que a temperatura na face externa do músculo maior do que a temperatura na face interna, e resfriamento, em que a temperatura na face externa do músculo menor do que a temperatura na face interna. O cálculo analítico e a simulação numérica foram executados para essas duas condições e os perfis de temperatura no interior da camada muscular, obtido por essas duas técnicas, foram comparados. 
O problema foi definido como unidimensional, em regime permanente, em coordenadas cartesianas e a temperatura interna do tecido muscular foi determinada como $37{ }^{\circ} \mathrm{C}$. Na Tabela 1 estão representadas as propriedades físicas, termofísicas e fisiológicas da camada muscular, utilizadas para os cálculos analíticos e para as simulações do problema. O detalhamento do domínio de simulação (modelo geométrico) é apresentado na Figura 1. O metabolismo $\mathrm{q}_{\mathrm{met}}\left(\mathrm{Wm}^{-3}\right)$, a perfusão sanguínea $w\left(\mathrm{~s}^{-1}\right)$, a temperatura arterial $\mathrm{T}_{\mathrm{a}}\left({ }^{\circ} \mathrm{C}\right)$, a condutividade térmica $\mathrm{k}\left(\mathrm{Wm}^{-1} \mathrm{~K}^{-1}\right)$ e as propriedades do sangue $\left[\mathrm{C}_{\mathrm{p}}\left(\mathrm{J} \mathrm{kg}^{-1} \mathrm{~K}^{-1}\right)\right.$ e $\left.\mathrm{r}\left(\mathrm{kgm}^{-3}\right)\right]$ foram considerados constantes.

Para a solução analítica desse problema simplificado, a equação de Pennes pode ser re-escrita como:

$\frac{d^{2} T}{d x^{2}}+\frac{q_{m e t}+\left(\rho c_{p} w\right)_{s}\left(T_{a}-T\right)}{k}=0$

A solução analítica correspondente, considerando valores conhecidos de temperatura nas extremidades da camada de tecido $\left[\mathrm{T}_{(\mathrm{x}=0 \mathrm{~m})}=\mathrm{T}_{\mathrm{i}}=37{ }^{\circ} \mathrm{C}\right.$ e $\mathrm{T}(\mathrm{x}=0,03 \mathrm{~m})=\mathrm{T}_{\mathrm{e}}$ ] é dada por (Incropera e Dewitt, 2008):

$\frac{\theta_{(x)}}{\theta_{(b)}}=\frac{\left(\frac{\theta_{(l)}}{\theta_{(b)}}\right) \operatorname{senh}(\bar{m} x)+\operatorname{senh}(\bar{m}(L-x))}{\operatorname{senh}(\bar{m} L)}$

em que: L representa a espessura total da camada muscular, os índices (b) e (l) correspondem a $\mathrm{x}=0$ e $\mathrm{x}=\mathrm{L}$, respectivamente, e:

$\theta=\frac{T-T_{a}-\dot{q}_{m e t}}{\left(\rho C_{p} \omega\right)_{b}}$

$\bar{m}^{2}=\frac{\left(\rho c_{p} w\right)_{b}}{k}$

As simulações unidimensionais foram realizadas para as situações de aquecimento e de resfriamento da articulação do joelho canino. Os resultados obtidos com as simulações foram avaliados e comparados com valores médios de temperatura obtidos a partir de um estudo experimental in vivo (Araújo, 2009). Nesse estudo, as temperaturas das estruturas peri e intra-articular do joelho foram mensuradas em dez cães. As temperaturas foram coletadas durante $40 \mathrm{~min}$ no caso do processo de aquecimento e, por $30 \mathrm{~min}$ no caso do resfriamento terapêutico. Para a realização das medidas, 10 termopares do tipo $\mathrm{K}$ convencional flexível foram utilizados.

As informações a respeito das fontes externas e do tempo utilizado para aquecer e resfriar o joelho canino, adotadas para a execução das simulações, também foram retiradas do estudo de Araújo (2009). Segundo Araújo (2009), uma manta térmica $(50 \mathrm{~cm} \times 29 \mathrm{~cm})$, com potência de saída de $35,2 \mathrm{~W}$, potência média de $31,2 \mathrm{~W}$ e controle automático de temperatura na faixa de $40{ }^{\circ} \mathrm{C}$ a $66^{\circ} \mathrm{C}$, programada para $66{ }^{\circ} \mathrm{C}$ e com temperatura média de $57,6^{\circ} \mathrm{C}$, mantida por $40 \mathrm{~min}$, foi utilizada para o aquecimento tecidual. $\mathrm{O}$ processo de resfriamento foi realizado por meio de duas compressas de gelo, cada uma confeccionada a partir de $1,0 \mathrm{~kg}$ de gelo manualmente triturado e com temperatura média de $4,5^{\circ} \mathrm{C}$, mantidas por $30 \mathrm{~min}$ (Araújo, 2009).

$\mathrm{O}$ problema físico unidimensional considerado envolveu o processo de transferência de calor na direção radial, em regime transiente, decorrente da aplicação de recursos térmicos de aquecimento e de resfriamento na articulação do joelho de um cão. $\mathrm{O}$ modelo geométrico do joelho canino, adotado como o domínio das simulações, foi definido por um cilindro composto por seis camadas teciduais, do exterior para o interior: a epiderme; o subcutâneo; o tecido adiposo; o tecido muscular; a região pericapsular e a cavidade intra-articular (que compreende o liquido sinovial e o osso). Na Figura 2 está representado o domínio unidimensional (modelo geométrico) da articulação do joelho utilizado para as simulações do aquecimento e resfriamento articular.

Os dados referentes à espessura, propriedades termofísicas a temperatura inicial $\left(\mathrm{T}_{\mathrm{i}}\right)\left({ }^{\circ} \mathrm{C}\right)$ de cada camada são apresentados na Tabela 2 . A camada mais externa da articulação (epiderme) $\left(\mathrm{r}_{\mathrm{e}}=42,62 \mathrm{~mm}\right)$ foi considerada em contato perfeito com as fontes de aquecimento e de resfriamento. Considerou-se a condição de simetria $(q=0)$ no centro da articulação.

Para o processo de aquecimento, foram efetuadas simulações com base nas seguintes condições de contorno na face externa da epiderme, estabelecidas com base em dados experimentais de Araújo (2009):

- Simulação A1 - temperatura da face externa equivalente à temperatura média da manta térmica $\left(57,6^{\circ} \mathrm{C}\right)$;

Tabela 1. Propriedades físicas, termofísicas e fisiológicas do sangue e da camada muscular, utilizadas para a verificação do software FEHT. Table 1. Physical, thermophysical and physiological properties for blood and muscle layer used for software verification.

\begin{tabular}{lccccccc}
\hline Camadas & $\begin{array}{c}\text { Espessura } \\
(\mathbf{m})\end{array}$ & $\begin{array}{c}\mathbf{k} \\
\left(\mathbf{W m} \mathbf{K}^{-1} \mathbf{K}\right)\end{array}$ & $\begin{array}{c}\mathbf{C}_{\mathrm{p}} \\
\left(\mathbf{J k g}^{-1} \mathbf{K}^{-1}\right)\end{array}$ & $\begin{array}{c}\rho \\
\left(\mathbf{k g m}^{-3}\right)\end{array}$ & $\begin{array}{c}\boldsymbol{W} \\
\left(\mathbf{s}^{-1}\right)\end{array}$ & $\begin{array}{c}\mathbf{q}_{\text {met }} \\
\left(\mathbf{W m}^{-3}\right)\end{array}$ & $\begin{array}{c}\mathbf{T}_{\mathbf{a}} \\
\left({ }^{\circ} \mathbf{C}\right)\end{array}$ \\
\hline Músculo & 0,03 & 0,5 & - & - & 0,0005 & 700 & - \\
Sangue & - & - & 3600 & 1000 & - & - & 37 \\
\hline
\end{tabular}

Fonte: Incropera e Dewitt (2008) 
- Simulação A2 - temperatura da face externa equivalente ao valor médio das medidas locais de temperatura após estabilização $\left(40,7^{\circ} \mathrm{C}\right)$;

- Simulação A3 - fluxo de calor na face externa equivalente ao fluxo médio de calor fornecido pela manta térmica e avaliado com base na razão entre a potência e área do dispositivo de aquecimento $\left(215,7 \mathrm{Wm}^{-2}\right)$.

Para o processo de resfriamento, foram efetuadas simulações com base nas seguintes condições de contorno na face externa da epiderme, estabelecidas com base em dados experimentais de Araújo (2009):

- Simulação R1 - temperatura da face externa equivalente à temperatura média das compressas de gelo $\left(4,5^{\circ} \mathrm{C}\right)$;

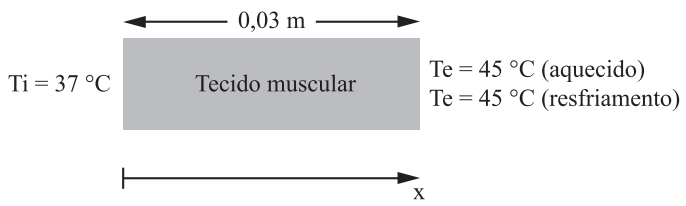

Figura 1. Modelo geométrico do problema unidimensional de biotransferência de calor utilizado para a verificação do software FEHT. Figure 1. Geometric model of the one-dimensional problem of bioheat transfer used to check the software FEHT.

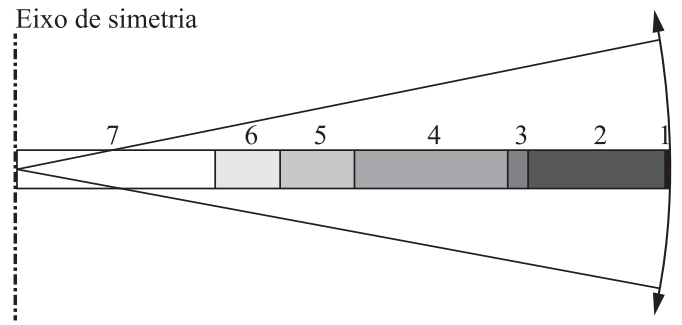

1 - Epiderme; 2 - Subcutâneo; 3 - Tecido adiposo;

4 - Músculo; 5 - Pericapsular; 6 - Líquido sinovial; 7 - Osso

Figura 2. Modelo geométrico da articulação do joelho canino utilizado para as simulações unidimensionais do aquecimento e do resfriamento terapêuticos.

Figure 2. Geometric model of the canine knee joint used for one-dimensional simulations of heating and cooling treatments.
- Simulação R2 - temperatura da face externa equivalente ao valor médio das medidas locais de temperatura após estabilização $\left(27,3{ }^{\circ} \mathrm{C}\right)$.

\section{Resultados e Discussão}

\section{Verificação do software}

No presente estudo, para a verificação do software FEHT duas situações distintas foram avaliadas para a camada de tecido muscular: 1) aquecimento - temperatura na face externa do músculo maior do que a temperatura na face interna $\left(\mathrm{T}_{\mathrm{e}}=45^{\circ} \mathrm{C}\right)$ e 2) resfriamento - temperatura na face externa do músculo menor do que a temperatura na face interna $\left(\mathrm{T}_{\mathrm{e}}=28,7^{\circ} \mathrm{C}\right)$.

A diferença percentual média entre os dados simulados e a solução analítica foi de apenas $0,0011 \%$ para a situação do aquecimento e de $0,0012 \%$ para a do resfriamento. Esses resultados confirmam que o software FEHT pode ser empregado na solução de problemas unidimensionais de biotransferência de calor.

\section{Simulação unidimensional}

Para a realização das simulações unidimensionais do aquecimento e do resfriamento foi gerada uma malha composta por 445 nós e 704 elementos. A temperatura média de cada camada simulada foi comparada com a respectiva temperatura experimental média. Exceção se faz à camada de tecido ósseo, cujos valores de temperatura não estavam disponíveis no estudo considerado para a comparação (Araújo, 2009). Desta forma, apenas os resultados da simulação dessa camada são apresentados.

\section{A-Aquecimento terapêutico}

$\mathrm{Na}$ Tabela 3 estão apresentadas as diferenças percentuais encontradas para os valores médios de temperatura obtidos para as simulações em comparação com os dados experimentais de Araújo (2009).

Tabela 2. Parâmetros físicos, termofísicos, fisiológicos e temperaturas iniciais dos tecidos utilizados nas simulações unidimensionais do aquecimento e do resfriamento.

Table 2. Physical, thermophysical, physiological parameters and initial temperatures of tissues used for one-dimensional simulations of heating and cooling.

\begin{tabular}{|c|c|c|c|c|c|c|c|}
\hline $\begin{array}{l}\text { Camada } \\
\text { tecidual }\end{array}$ & $\begin{array}{l}\text { Espessura } \\
\text { (m) }\end{array}$ & $\begin{array}{c}\mathbf{c}_{\mathrm{p}} \\
\left(\mathrm{Jkg}^{\left.-{ }^{\mathrm{O}} \mathrm{C}^{-1}\right)}\right.\end{array}$ & $\begin{array}{c}\rho \\
\left(\mathrm{kgm}^{-3}\right)\end{array}$ & $\begin{array}{c}\mathbf{k} \\
\left(\mathbf{W m}^{-10} \mathbf{C}^{-1}\right)\end{array}$ & $\begin{array}{c}\mathbf{q}_{\mathbf{m}} \\
\left(\mathbf{W} \mathbf{m}^{-3}\right)\end{array}$ & $\begin{array}{c}\mathbf{W} \\
\left(\mathbf{m}^{3} \mathbf{s}^{-1} \mathbf{m}^{-3} \text { tec }\right)\end{array}$ & $\mathbf{T}_{\mathbf{i}}\left({ }^{\circ} \mathrm{C}\right)$ \\
\hline Epiderme & $8,0 \times 10^{-5}$ & 3593 & 1200 & $2,28 \times 10^{-1}$ & 0 & 0 & 34,9 \\
\hline Subcutâneo & $9,0 \times 10^{-3}$ & 3365 & 1200 & $4,64 \times 10^{-1}$ & 200 & $1,3 \times 10^{-3}$ & 35,5 \\
\hline Tecido adiposo & $1,3 \times 10^{-3}$ & 2678 & 937 & $2,03 \times 10^{-1}$ & 3,9 & $2,9 \times 10^{-4}$ & 36,1 \\
\hline Tecido muscular & $1,0 \times 10^{-2}$ & 3684 & 1097 & $5,29 \times 10^{-1}$ & 716 & $5,8 \times 10^{-4}$ & 36,5 \\
\hline Pericapsular & $4,9 \times 10^{-3}$ & 3500 & 1051 & $4,98 \times 10^{-1}$ & 0 & $1,8 \times 10^{-3}$ & 35,2 \\
\hline Líquido sinovial & $4,2 \times 10^{-3}$ & 4190 & 1000 & $6,10 \times 10^{-1}$ & 0 & 0 & 37,1 \\
\hline Osso & $1,3 \times 10^{-2}$ & 1785 & 1585 & $7,35 \times 10^{-1}$ & 368,3 & $4,0 \times 10^{-4}$ & 37,1 \\
\hline Sangue & - & 3813 & 1038 & - & - & - & 37,0 \\
\hline
\end{tabular}


Tabela 3. Diferença percentual média encontrada entre os valores médios de temperatura experimental e das simulações A1, A2 e A3, durante o processo de aquecimento, para as camadas teciduais que compõem o joelho canino.

Table 3. Average percentage difference (\%) found between the mean temperature of the experiment and the simulations A1, A2 and A3, during heating, for the layers that compose the canine knee joint.

\begin{tabular}{lccc}
\hline \multirow{2}{*}{ Camada } & \multicolumn{3}{c}{ Diferença percentual média (\%) } \\
\cline { 2 - 4 } & $\begin{array}{c}\text { Simulação } \\
\text { A1 }\end{array}$ & $\begin{array}{c}\text { Simulação } \\
\text { A2 }\end{array}$ & $\begin{array}{c}\text { Simulação } \\
\text { A3 }\end{array}$ \\
\hline Epiderme & 36,7 & - & 1,6 \\
Subcutâneo & 27,3 & 1,8 & 2,4 \\
Tecido adiposo & 14,7 & 0,4 & 0,2 \\
Músculo & 6,7 & 0,7 & 2,3 \\
Pericapsular & 1,9 & 1,1 & 2,8 \\
Líquido sinovial & 1,7 & 1,2 & 3,0 \\
Média & 14,8 & 1,0 & 2,1 \\
\hline
\end{tabular}

Observa-se que houve uma diferença significativa entre os resultados de simulação e experimentais para a situação em que a temperatura da face externa da epiderme foi equivalente à temperatura média da manta térmica (simulação A1). Os valores de temperatura obtidos na simulação foram superiores aos valores experimentais, sendo esta diferença mais significativa para as camadas mais externas. Tal comportamento é atribuído ao fato de que a consideração de temperatura da face externa equivalente à temperatura média da manta térmica corresponde a se desprezar a resistência térmica da manta. Observa-se que houve uma melhor concordância entre experimentos e simulação quando o valor médio da temperatura externa da epiderme foi utilizado como condição de contorno (simulação A2) ou quando a condição de contorno foi baseada na consideração de que o fluxo de calor na face externa era equivalente ao fluxo médio de calor fornecido pela manta térmica (simulação A3).

As variações da temperatura média das diversas camadas de tecido durante o aquecimento, referentes às simulações $\mathrm{A} 2$ e $\mathrm{A} 3$, podem ser observadas nas Figuras 3 e 4, respectivamente. Uma análise da Figura 3 indica que, nos instantes iniciais do processo de aquecimento, nas camadas mais superficiais (epiderme, subcutâneo e tecido adiposo), a temperatura simulada aumenta mais rapidamente em comparação com as medidas experimentais. Vale ressaltar que o valor de temperatura utilizado como condição de contorno não considerou a variação de temperatura que ocorre durante os primeiros $10 \mathrm{~min}$ de aquecimento. Durante este intervalo, a temperatura média externa da epiderme aumentou de 34,9 a $39,6^{\circ} \mathrm{C}$. A diferença também pode ser associada ao fato de que o modelo não considera as variações reais que ocorrem nos parâmetros fisiológicos em decorrência do aquecimento tais como a dilatação de vasos sanguíneos e consequente aumento da perfusão sanguínea, que diminui a taxa de aquecimento.
$\mathrm{Na}$ Figura 3c pode-se notar que o tecido adiposo mantém a sua temperatura inicial inalterada nos primeiros instantes da simulação. Apesar desse comportamento, denominado como "tempo morto", não ser evidenciado na curva experimental média do estudo de Araújo (2009), segundo a autora, ele foi observado em alguns animais participantes do estudo. De acordo com Chaui-Berlinck et al. (2005), o atraso da resposta tecidual a um estímulo térmico é uma característica dos sistemas biológicos. Isso pode ser explicado pelo fato da pele, do subcutâneo e, em especial, do tecido adiposo constituírem um isolante térmico para as estruturas internas do corpo. Essas estruturas, por apresentarem uma alta resistência térmica, limitam a transferência de calor entre o meio externo e o interno (Pardasani e Adlakha, 1995).

O comportamento da temperatura das camadas teciduais obtido com a simulação baseada na consideração de que a epiderme recebeu todo o fluxo de calor proveniente da manta térmica pode ser visto na Figura 4. Os valores médios correspondentes às diferenças entre temperaturas simuladas e medidas foram similares aos obtidos na simulação A2. No entanto, a simulação A3 mostrou-se mais adequada à descrição do fenômeno, pois independe do conhecimento prévio de valores experimentais da temperatura externa da epiderme. As diferenças entre experimentos e simulações podem estar associadas ao fato de que na simulação não se considerou a perda de calor da manta para o ar ambiente.

Os perfis de temperatura obtidos para a simulação A3, nos instantes iniciais (60 s) e finais (2340 s), estão apresentados na Figura 5. A comparação das duas curvas demonstra que houve uma elevação da temperatura de todos os tecidos avaliados. Entretanto, o aumento da temperatura foi maior nos tecidos superficiais em comparação aos tecidos profundos. Esse fato vai de encontro com a literatura, que relata que os tecidos profundos reagem ao aquecimento por condução de maneira semelhante aos tecidos superficiais, porém em menor intensidade (Low e Reed, 2001). Segundo Pardasani e Adlakha (1995) a pele, o subcutâneo e o tecido adiposo funcionam como uma barreira isolante do corpo e influenciam, portanto, no grau de variação da temperatura dos tecidos internos. Isso pode ser observado, também, através dos elevados gradientes de temperatura do subcutâneo e do tecido adiposo em comparação com os demais tecidos.

\section{B - Resfriamento terapêutico}

Apresenta-se na Tabela 4 a comparação entre os valores médios de temperatura obtidos para as simulações em comparação com os dados experimentais de 

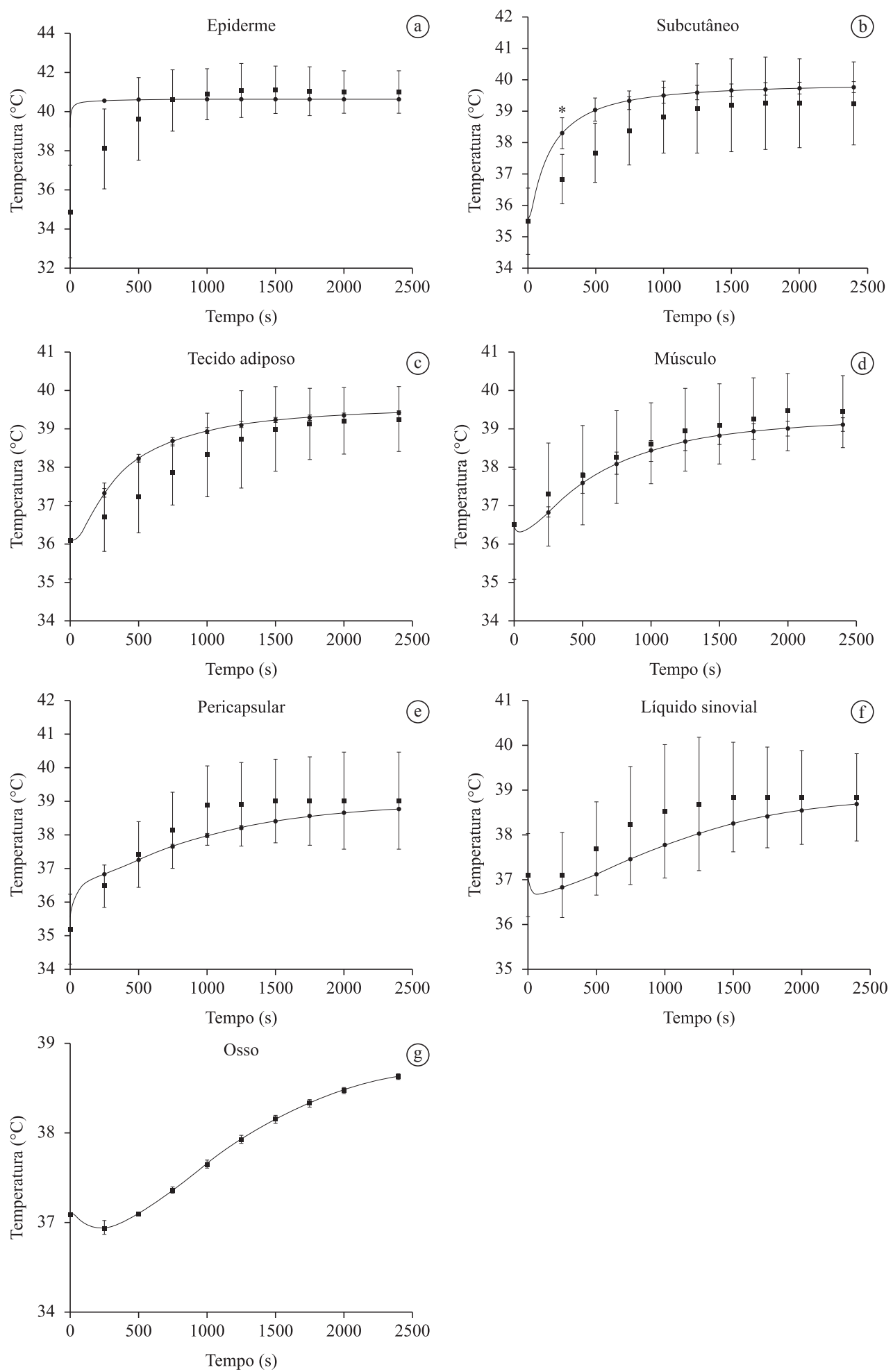

Figura 3. Comportamento da temperatura média das camadas que compõem a articulação do joelho canino: a) epiderme, b) subcutâneo, c) tecido adiposo, d) músculo, e) pericapsular, f) líquido sinovial e g) osso, durante o aquecimento - dados experimentais; - simulação A2. As barras de erro correspondem aos valores de desvio padrão. Não foram detectadas diferenças significativas entre os valores médios experimentais e de simulação (Teste de Tukey, $\mathrm{p}>0,05$ ).

Figure 3. Behavior of the average temperature of the canine knee layers: a) epidermis, b) subcutaneous, c) fat, d) muscle, e) pericapsular, f) synovial liquid and g) bone during heating - experiment; - simulation A2. Error bars represent the standard deviation values. There were not significant differences between experimental and simulation average values (Tukey test, $p>0.05$ ). 

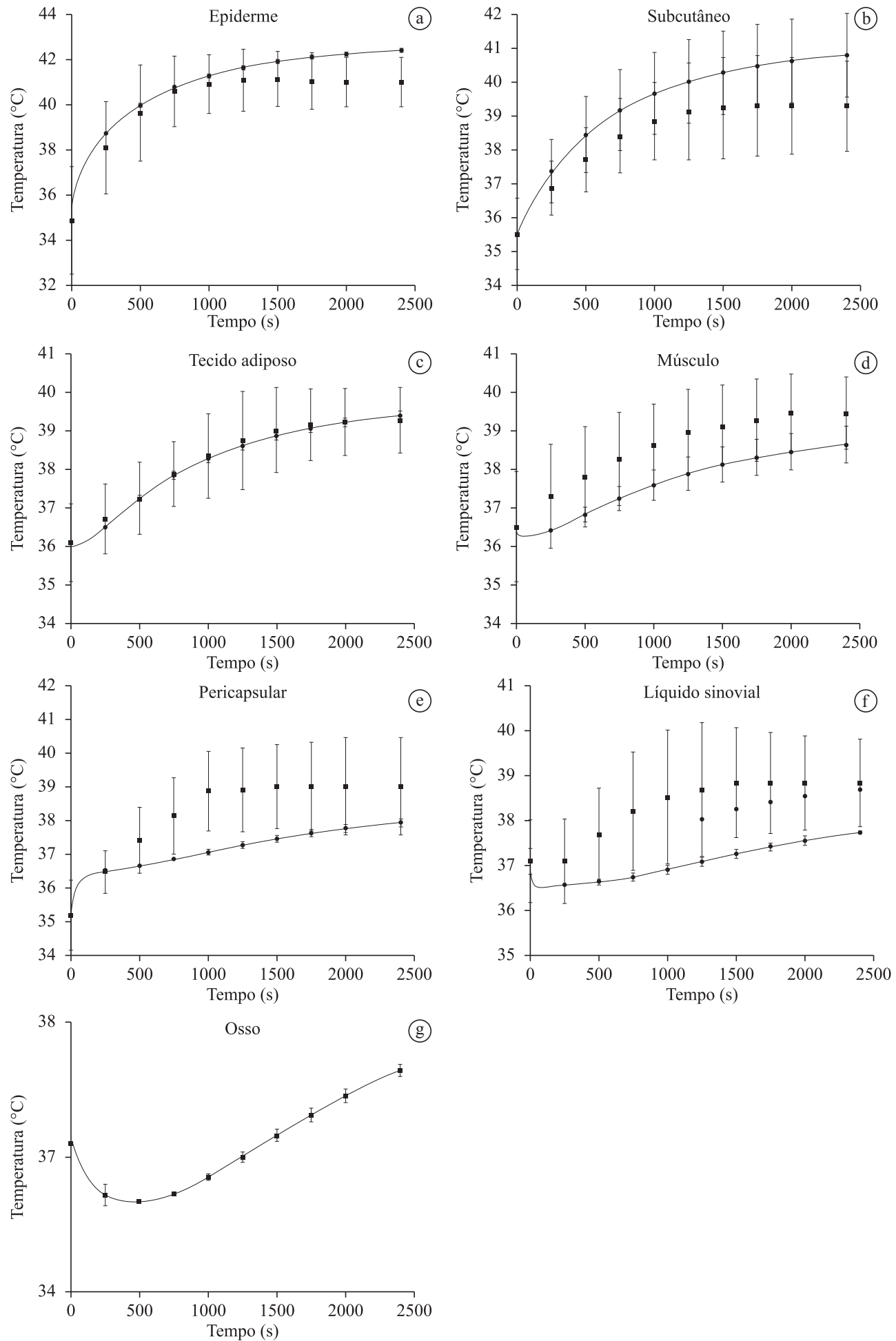

(g)

Figura 4. Comportamento da temperatura média das camadas que compõem a articulação do joelho canino: a) epiderme, b) subcutâneo, c) tecido adiposo, d) músculo, e) pericapsular, f) líquido sinovial e g) osso, durante o aquecimento - a dados experimentais; - simulação A3. As barras de erro correspondem aos valores de desvio padrão. Não foram detectadas diferenças significativas entre os valores médios experimentais e de simulação (Teste de Tukey, $\mathrm{p}>0,05$ ).

Figure 4. Behavior of the average temperature of the canine knee layers: a) epidermis, b) subcutaneous, c) fat, d) muscle, e) pericapsular, f) synovial liquid and g) bone during heating - experiment; - simulation A3. Error bars represent the standard deviation values. There were not significant differences between experimental and simuation average values (Tukey test, $p>0.05$ ). 


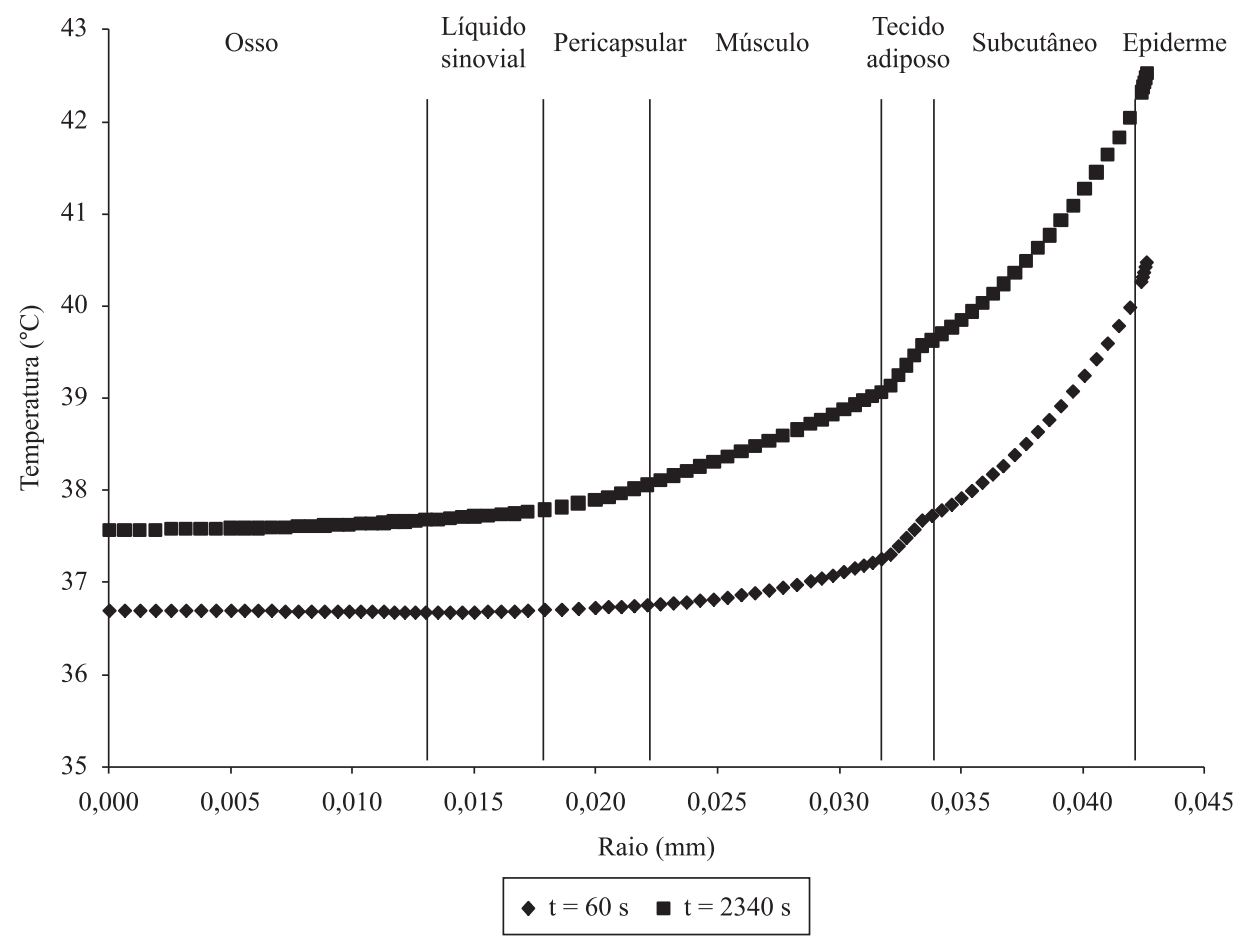

Figura 5. Perfis de temperatura ao longo do raio das camadas epiderme, subcutâneo, tecido adiposo, músculo, pericapsular, líquido sinovial e osso nos instantes iniciais (60 s) e finais (2340 s) da simulação A3.

Figure 5. Temperature profiles along the radius of the layers epidermis, subcutaneous, fat, muscle, pericapsular, synovial liquid and bone at the beginning (60 s) and end (2340 s) of simulation $\mathrm{A} 3$.

Tabela 4. Diferença percentual média (\%) encontrada entre os valores médios de temperatura experimental e das simulações R1 e R2, durante o processo de resfriamento, para as camadas teciduais que compõem o joelho canino.

Table 4. Average percentage difference (\%) found between the mean temperature of the experiment and the simulations $R 1$ and $R 2$, during cooling, for the layers that compose the canine knee joint.

\begin{tabular}{lcc}
\hline \multirow{2}{*}{ Camada } & \multicolumn{2}{c}{ Diferença percentual média (\%) } \\
\cline { 2 - 3 } & Simulação R1 & Simulação R2 \\
\hline Epiderme & 36,1 & - \\
Subcutâneo & 27,5 & 7,3 \\
Tecido adiposo & 11,0 & 16,3 \\
Músculo & 5,6 & 23,3 \\
Pericapsular & 15,8 & 25,2 \\
Líquido sinovial & 6,6 & 12,9 \\
Média & 17,1 & 17,0 \\
\hline
\end{tabular}

Araújo (2009). Observam-se diferenças significativas entre os resultados de simulação e experimentais, tanto para a situação em que a temperatura da face externa da epiderme foi equivalente à temperatura média da manta térmica (simulação R1) como para a situação em que o valor médio experimental da temperatura da face externa da epiderme foi utilizado como condição de contorno (simulação R2). As diferenças foram mais significativas para as camadas mais externas, no caso da simulação R1. Tal fato é decorrente do erro associado a se desprezar a resistência térmica da compressa de gelo, incorrendo em um valor de temperatura na superfície externa significativamente inferior ao valor experimental. A utilização do valor médio experimental da temperatura da face externa como condição de contorno promoveu uma diminuição do erro com relação à temperatura média da camada de tecido subcutâneo, porém o erro aumentou para as camadas mais internas.

O comportamento da temperatura das camadas teciduais durante o processo de resfriamento, baseada no pressuposto que a temperatura da face externa da epiderme era igual à temperatura média da compressa de gelo $\left(4,5^{\circ} \mathrm{C}\right)$, está representado na Figura 6 . Observa-se que as temperaturas simuladas foram significativamente subestimadas para as camadas mais externa (epiderme e subcutâneo). Observa-se ainda que, embora as temperaturas simuladas para as demais camadas encontrem-se, em sua maioria, dentro da faixa de erro das medidas experimentais, a descrição do comportamento não é adequada, com a temperatura dos tecidos superficiais inferior ao recomendado pela literatura para que danos térmicos aos tecidos não 

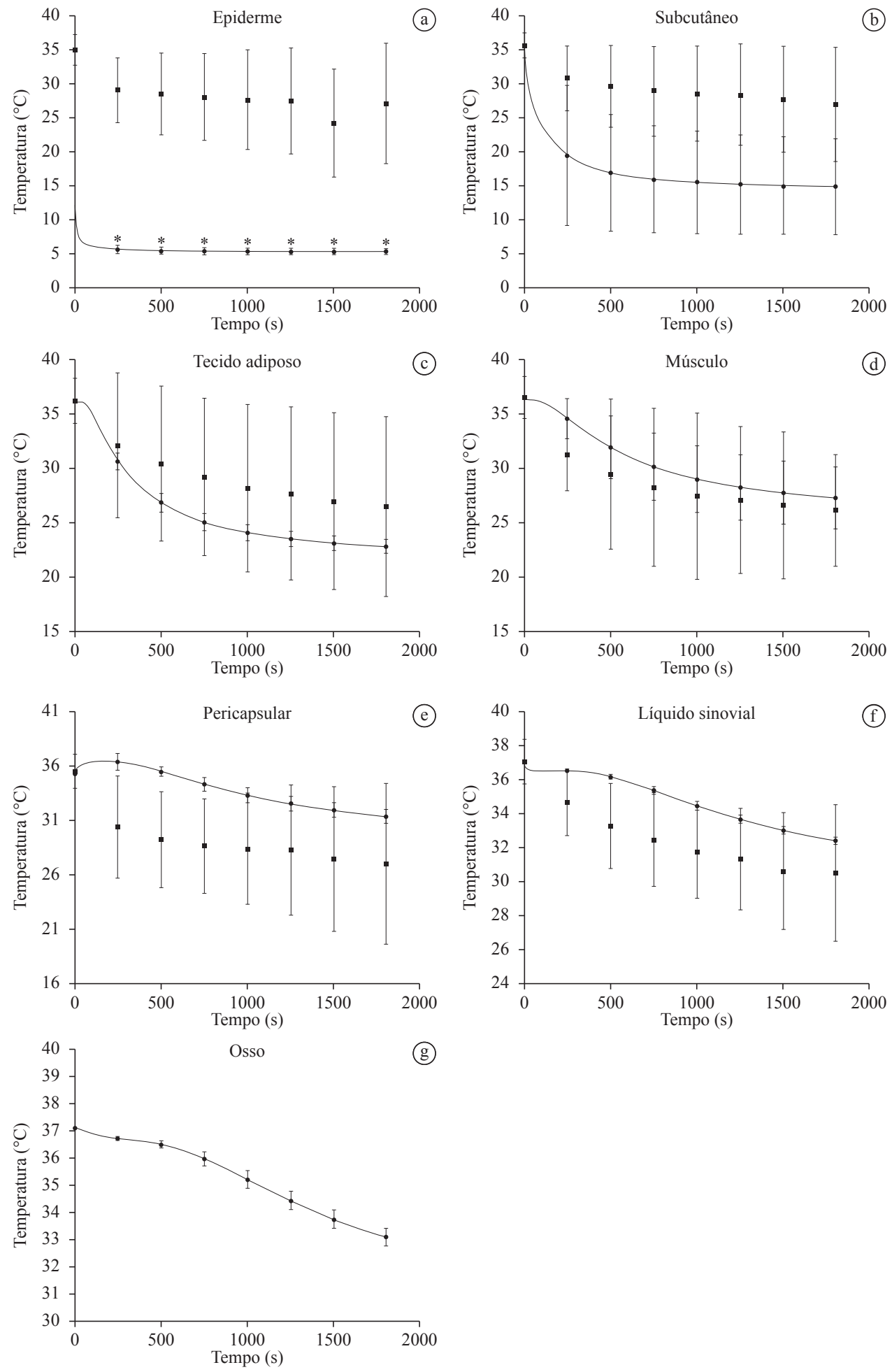

Figura 6. Comportamento da temperatura média das camadas que compõem a articulação do joelho canino: a) epiderme, b) subcutâneo, c) tecido adiposo, d) músculo, e) pericapsular, f) líquido sinovial e g) osso, durante o resfriamento - - dados experimentais; - simulação R1. As barras de erro correspondem aos valores de desvio padrão. Médias destacadas com asteriscos $(*)$ nas figuras apresentaram diferença significativa com base em análise de variância $(\mathrm{p}>0,05)$.

Figure 6. Behavior of the average temperature of the canine knee layers: a) epidermis, b) subcutaneous, c) fat, d) muscle, e) pericapsular, f) synovial liquid and g) bone during cooling - experiment; - simulation R1.Error bars represent standard deviation values. Average values marked with an asterisk (*) differ significantly (Tukey test, $p>0.05$ ). 
ocorram $\left(10^{\circ} \mathrm{C}\right)$ e um pequeno aumento de temperatura durante a simulação (tecido pericapsular). Tal aumento está provavelmente associado ao efeito do calor gerado em decorrência do metabolismo, uma vez que nos instantes iniciais este efeito não é contraposto pelo resfriamento para as camadas mais internas.

Dentre as simulações realizadas no presente estudo, as simulações do aquecimento forneceram uma melhor descrição dos dados experimentais. Dentre as condições de contorno avaliadas no presente trabalho, a condição de fluxo de calor foi mais adequada para representar a transferência de calor na articulação. Apesar dessa condição também não levar em consideração as perdas de calor para o ambiente, $o$ fato do ar não ser um bom condutor minimizaria o impacto dessa perda. Também é válido ressaltar que as medidas experimentais estão sujeitas a erros devido à dificuldade de controle de diversos parâmetros. Segundo Gonçalves Júnior e Sousa (2008), para que uma medição seja realizada sem erros, seriam necessários: 1) um sistema de medição perfeito; 2) um ambiente controlado e perfeitamente estável; 3) um operador perfeito e 4) que a grandeza medida tivesse um valor único, perfeitamente definível e estável. Entretanto, na prática não é comum essas condições acontecerem isoladamente e muito menos simultaneamente. Desta forma, em menor ou maior escala, sempre haverá um erro de medição, o qual pode justificar as diferenças encontradas.

Os parâmetros termofísicos (condutividade térmica, calor específico e etc.) e fisiológicos (metabolismo e perfusão sanguínea), vinculados ao processo de biotransferência de calor, são bastante discrepantes na literatura. Essas diferenças demonstram que ainda existem incertezas na medida experimental desses parâmetros. Desta forma, a aproximação equivocada de qualquer um dos parâmetros utilizados também pode ter sido um dos fatores responsáveis pelas diferenças encontradas. Segundo Cui e Barbenel (1990 e 1991) dos parâmetros fisiológicos e termofísicos, a perfusão sanguínea e a condutividade térmica são os que mais influenciam o comportamento e as respostas térmicas. Elas têm influência importante no comportamento isolante dos tecidos, no tempo para se alcançar o equilíbrio e nos diferentes valores no perfil de temperatura da região avaliada (Lima et al., 2006).

Desta forma, apesar de algumas diferenças encontradas as simulações realizadas com o software FEHT podem ser um importante passo para a avaliação não invasiva da eficácia dos recursos térmicos. Adicionalmente, as simulações do presente trabalho também podem ser utilizadas para fins didáticos, uma vez que a falta de conhecimento sobre as respostas térmicas dos tecidos alvos e as divergências de informações na literatura têm feito com que os recursos termoterápicos sejam erroneamente utilizados e consequentemente desprezados pelos profissionais da fisioterapia.

\section{Conclusão}

Com o trabalho realizado foi possível simular a transferência de calor na articulação do joelho canino durante o aquecimento e o resfriamento terapêuticos. Os resultados encontrados no presente estudo demonstram que tanto a simulação A2, quanto a simulação A3 foram capazes de representar adequadamente o processo de aquecimento tecidual. No entanto, a simulação A3 mostrou-se mais adequada à descrição do fenômeno, pois independe do conhecimento prévio de valores experimentais da temperatura externa da epiderme. Em contrapartida, as simulações do resfriamento tecidual (simulação R1 e R2) não apresentaram boa aproximação com os dados experimentais, sugerindo uma maior complexidade de avaliação desse processo.

As simulações realizadas no presente estudo são o primeiro passo para uma melhor compreensão não invasiva do comportamento da temperatura dos tecidos durante a aplicação de recursos físicos de aquecimento e resfriamento. A fim de aprimorar os resultados obtidos, simulações bidimensionais, considerando variações reais da geometria da articulação do joelho canino, serão efetuadas em trabalhos futuros.

\section{Agradecimentos}

Os autores agradecem ao $\mathrm{CNPq}$ pelo auxílio financeiro concedido (Processo 554666/2009-0).

\section{Referências}

Araújo AR. Formulação e validação de um modelo térmico para estimativa da temperatura intra-articular [tese]. Belo Horizonte: Universidade Federal de Minas Gerais; 2009.

Chaui-Berlinck JG, Navas CA, Monteiro LHA, Bicudo JEPW. Control of metabolic rate is a hidden variable in the allometric scaling of homeotherms. The Journal of Experimental Biology. 2005; 208:1709-16. PMid:15855402. http://dx.doi.org/10.1242/jeb.01421

Chesterton LS, Foster NE, Ross L. Skin temperature response to cryotherapy. Archives of Physical Medicine \& Rehabilitation. 2002; 83:543-9. PMid:11932859. http://dx.doi.org/10.1053/apmr.2002.30926

Cui ZF, Barbenel CJ. The influence of model parameter values on the prediction of skin surface temperature: I. Resting and surface insulation. Physics in Medicine and Biology. 1990; 35(12):1683-97. PMid:2284337. http://dx.doi.org/10.1088/0031-9155/35/12/007

Cui ZF, Barbenel CJ. The influence of model parameter values on the prediction of skin surface temperature: II. Contact problems. Physics in Medicine and 
Biology. 1991; 36(12):1607-20. PMid:1771183. http://dx.doi.org/10.1088/0031-9155/36/12/006

Deal DN, Tipton J, Rosencrance E, Curl WW, Smith TL. Ice reduces edema. The Journal of Bone \& Joint Surgery. 2002; 84(9):1573-8.

Doherty CB, Doherty SD, Rosen T. Thermotherapy in dermatologic infections. Journal of the American Academy of Dermatology. 2009; 62:909-27. PMid:20466169. http://dx.doi.org/10.1016/j.jaad.2009.09.055

Draper DO, Knight K, Fujiwara T, Castel JC. Temperature change in human muscle during and after pulsed shortwave diathermy. Journal of Orthopaedic \& Sports Physical Therapy. 1999; 29(1):13-22. PMid:10100117.

Gonçalves Júnior AA, Sousa AR. Fundamentos de metrologia científica e industrial. São Paulo: Manole; 2008.

Greenstein G. Therapeutic efficacy of cold therapy after intraoral surgical procedures: a literature review. Journal of Periodontology. 2007; 78(5):790-800. PMid:17470011. http://dx.doi.org/10.1902/jop.2007.060319

Incropera F, Dewitt DP. Fundamentos de transferência de calor e massa. 6th ed. Rio de Janeiro: LTC Editora; 2008.

Karaa S, Zhang J, Yang F. A numerical study of a 3D bioheat transfer problem with different spatial heating. Mathematics and Computers in Simulation. 2005; 68:375-88. http://dx.doi.org/10.1016/j.matcom.2005.02.032

Kennet J, Hardaker N, Hobbs S, Selfe J. Cooling efficiency of 4 common cryotherapeutic agents. Journal of Athletic Training. 2007; 42(3):343-8.

Knight CA, Rutledge CR, Cox ME, Acosta M, Hall SJ. Effect of superficial heat, deep heat, and active exercise warm-up on the extensibility of the plantar flexors. Physical Therapy. 2001; 81(6):1206-14.

Lima RCF, Lyra PRM, Guimarães CSC, Carvalho DKE, Silva GMLL. Modelagem computacional da biotransferência de calor no tratamento por hipertermia em tumores de duodeno através do método dos volumes finitos em malhas não estruturadas. Revista Brasileira de Engenharia Biomédica. 2006; 22(2):119-29.
Low J, Reed A. Eletroterapia explicada: princípios e prática. 3th ed. São Paulo: Manole; 2001.

Maggi LE, Omena TP, von Kruger MA, Pereira WCA. Software didático para modelagem do padrão de aquecimento dos tecidos irradiados por ultra-som fisioterapêutico. Revista Brasileira de Fisioterapia. 2008; 12(3):204-14. http://dx.doi. org/10.1590/S1413-35552008000300008

Nadler AF, Weingand K, Kruse RJ. The Physiologic Basis and Clinical Applications of Cryotherapy and Thermotherapy for the Pain Practitioner. Pain Physician. 2004; 7:395-9.

Pardasani KR, Adlakha N. Coaxial circular sector elements to study two dimensional heat distribution problem in dermal regions of humam limbs. Mathematical and Computer Modelling. 1995; 22(9):127-140. http://dx.doi. org/10.1016/0895-7177(95)00173-Y

Pennes HH. Analysis of tissue and arterial blood temperatures in the resting human forearm. Journal of Applied Physiology. 1948; 1(2):93-122.

Robertson VJ, Ward AR, Jung P. The effect of heat on tissue extensibility: a comparison of deep and superficial heating. Archives of Physical Medicine \& Rehabilitation. 2005; 86:819-25. PMid:15827938. http://dx.doi.org/10.1016/j.apmr.2004.07.353

Shrivastava D, Vaughan JT. A generic bioheat transfer thermal model for a perfused tissue. Journal of Biomechanical Engineering. 2009; 131(7):1-12. PMid:19640142. PMCid:2737815. http://dx.doi.org/10.1115/1.3127260

Trobec R, Sterk M, Almawerd S, Veselko M. Computer simulation of topical knee cooling. Computers in Biology and Medicine. 2008; 38:1076-83. PMid:18823623. http://dx.doi.org/10.1016/j.compbiomed.2008.08.004

Zhang W, Moskowitz RW, Nuki G, Abramson S, Altman $\mathrm{RD}$, Arden N, et al. OARSI recommendations for the management of hip and knee osteoarthritis, Part II: OARSI evidence-based, expert consensus guidelines. Osteoarthritis and Cartilage. 2008; 16(2):137-62. PMid:18279766. http://dx.doi.org/10.1016/j.joca.2007.12.013

\section{Autores}

